\title{
Dietary Supplementation with Raspberry Extracts Modifies the Fecal Microbiota in Obese Diabetic $\mathrm{db} / \mathrm{db}^{\mathrm{b}}$ Mices
}

\author{
Jose F. Garcia-Mazcorro ${ }^{1}$, Romina Pedreschi ${ }^{2}$, Boon Chew ${ }^{3}$, Scot E. Dowd ${ }^{4}$, Jorge R. Kawas ${ }^{5}$, and Giuliana Noratto ${ }^{3 *}$ \\ ${ }^{1}$ Research and Development, MNA de México, San Nicolás de los Garza 66477, México \\ ${ }^{2}$ School of Agronomy, Pontificia Universidad Católica de Valparaíso, La Palma 2260000, Chile \\ ${ }^{3}$ Department of Nutrition and Food Science, Texas AEM University, College Station, TX 77843, USA \\ ${ }^{4}$ Molecular Research LP, Shallowater, TX 79363, USA \\ ${ }^{5}$ Faculty of Agronomy, Universidad Autónoma de Nuevo León, General Escobedo 66050, México
}

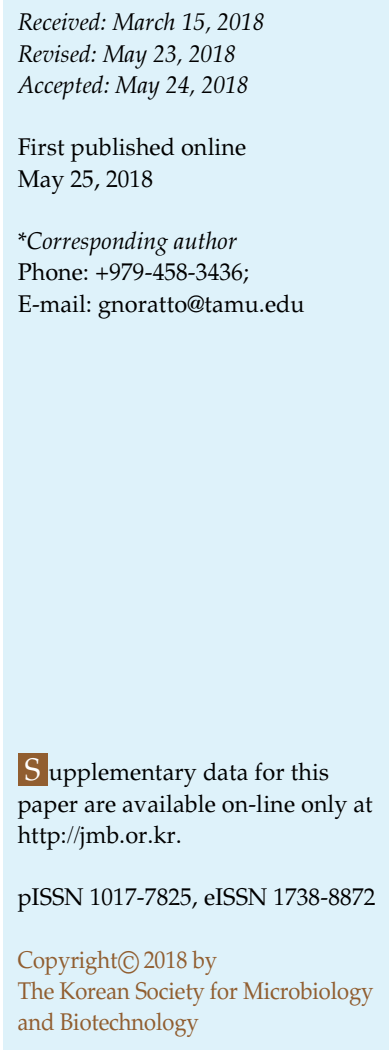

S upplementary data for this paper are available on-line only at http://jmb.or.kr.

pISSN 1017-7825, eISSN 1738-8872

Copyright(C) 2018 by

The Korean Society for Microbiology and Biotechnology

Raspberries are polyphenol-rich fruits with the potential to reduce the severity of the clinical signs associated with obesity, a phenomenon that may be related to changes in the gut microbiota. The aim of this study was to investigate the effect of raspberry supplementation on the fecal microbiota using an in vivo model of obesity. Obese diabetic $\mathrm{db} / \mathrm{db}$ mice were used in this study and assigned to two experimental groups (with and without raspberry supplementation). Fecal samples were collected at the end of the supplementation period (8 weeks) and used for bacterial 16S rRNA gene profiling using a MiSeq instrument (Illumina). QIIME 1.8 was used to analyze the $16 \mathrm{~S}$ data. Raspberry supplementation was associated with an increased abundance of Lachnospiraceae $(p=0.009)$, a very important group for gut health, and decreased abundances of Lactobacillus, Odoribacter, and the fiber degrader S24-7 family as well as unknown groups of Bacteroidales and Enterobacteriaceae $(p<0.05)$. These changes were enough to clearly differentiate bacterial communities accordingly to treatment, based on the analysis of UniFrac distance metrics. However, a predictive approach of functional profiles showed no difference between the treatment groups. Fecal metabolomic analysis provided critical information regarding the raspberry-supplemented group, whose relatively higher phytosterol concentrations may be relevant for the host health, considering the proven health benefits of these phytochemicals. Further studies are needed to investigate whether the observed differences in microbial communities (e.g., Lachnospiraceae) or metabolites relate to clinically significant differences that can prompt the use of raspberry extracts to help patients with obesity.

Keywords: Raspberry, polyphenols, obesity, gut microbiota

\section{Introduction}

Obesity is a serious problem affecting millions of people worldwide [1]. Obesity is associated with a higher risk for depression, heart disease, cardiopulmonary disease, stroke, diabetes, cancer, and hypertension [2], and it represents a significant economic burden to both individuals and society as a whole [3]. Fortunately, in most cases, obesity can be prevented and/or treated. For example, certain dietary patterns and common weight-loss diets can be effective for weight loss, glycemic control, and cardiovascular risk factors [4].

The digestive tract of humans and animals is a muscular canal that allows the uptake of nutrients that are indispensable for fulfilling the body's metabolic needs. This organ is populated by millions of different microorganisms (the gut microbiota) that play a pivotal role in gut health [5], behavior [6], and immunity [7]. The gut microbiota is normally in equilibrium with the host, but this symbiosis can be disrupted during disease, such as gut inflammation 
and obesity [8]. Importantly, growing evidence shows that a healthy gastrointestinal environment is dependent on factors that affect the microbiota, such as dietary patterns $[9,10]$.

Red raspberries are fruits containing bioactive polyphenols such as ellagitannins and anthocyanins [11] that have a potential to modify the body metabolism, therefore modulating health in patients suffering with weight disorders [12]. The health benefits associated with these substances are various and relate in part to the inhibition of digestive enzymes [13], a decrease of oxidative stress [14], and a change in the gut microbiota [15]. Ellagitannins from red raspberry exert cytotoxic effects against human colon adenocarcinoma in vitro [16] and reduce secondary bile acids in rats fed a high-fat diet [17]. Other fruits such as pomegranate also contain ellagitannins, and this fruit has been shown to have anti-inflammatory and antitumoral properties [18], to promote a protective effect in obesityassociated nonalcoholic fatty liver disease [19], to help ameliorate metabolic syndrome [20], and to reduce thiobarbituric acid reactive substances in overweight subjects [21]. The gut microbiota is capable of metabolizing polyphenols [22] and this phenomenon is partly responsible for the effect of these substances in gut health, microbial composition, and metabolic activity [15, 23-28]. This body of research prompts investigating the effect of polyphenolreach dietary ingredients on the gut microbiota. The objective of this study was to evaluate the effect of raspberry supplementation on the gut microbiota of obese mice.

\section{Materials and Methods}

\section{Ethical Approval}

All experimental procedures were approved by the Ethics Committee at the University of Washington (IACUC No. 04436001).

\section{Raspberry}

Wakefield red raspberries grown in 2014 in Washington State were harvested at a mature stage $(10.7 \%$ total soluble solids, maturity index of 6.5 , measured as soluble solids divided by titratable acidity) and individually quick-frozen. Frozen raspberries were kindly supplied by Endfield Farms and by Rader Farms, Inc. (USA). The raspberries were freeze dried at $-20^{\circ} \mathrm{C}$ and $40 \mathrm{mTorr}$ pressure using a freeze dryer (VirTis Co., USA) and stored at $-80^{\circ} \mathrm{C}$ for use in diet preparation.

\section{Animals, Diets, and Experimental Design}

Leptin receptor-deficient $\mathrm{db} / \mathrm{db}$ male mice (BKS.Cg-Dock7m $+/+$ Leprdb/OlaHsd) were used as the in vivo model of obesity
Table 1. AIN-93G diet composition.

\begin{tabular}{lcccc}
\hline \multirow{2}{*}{ Ingredient } & \multicolumn{2}{c}{ Control } & \multicolumn{2}{c}{ Raspberry } \\
\cline { 2 - 5 } & Weight $(\mathrm{g})$ & kcal & Weight $(\mathrm{g})$ & kcal \\
\hline Casein & 100 & 400 & 100 & 400 \\
Maltodextrin & 66 & 264 & 66 & 264 \\
Sucrose & 50 & 200 & 50 & 200 \\
Cellulose & 25 & 0 & 25 & 0 \\
Mineral mix & & & & \\
Vitamin mix & 17.5 & 0 & 17.5 & 0 \\
L-Cysteine & 5 & 0 & 5 & 0 \\
Choline bitartrate & 1.5 & 6 & 1.5 & 6 \\
$t$-Butylhydroquinone & 0.007 & 0 & 0.007 & 0 \\
Cornstarch & 198.75 & 795 & 150 & 600 \\
Soybean oil & 35 & 315 & 35 & 315 \\
Raspberry & 0 & 0 & 48.75 & 195 \\
Agar & 20 & 0 & 20 & 0 \\
Water & 480 & 0 & 480 & 0 \\
Total & $1,000.01$ & 1,980 & $1,000.01$ & 1,980 \\
\hline
\end{tabular}

${ }^{a, b}$ For the mineral and vitamin mix, please refer to Supplementary Table S1.

${ }^{c}$ Final raspberry supplementation was $5.3 \%$ due to water evaporation during agar-based diet preparation ( $8 \%)$. Raspberry contributed with polyphenolics (963 mg extractable GAE/kg agar-based diet and $111 \mathrm{mg}$ non-extractable PA/kg agar-based diet) and $18.6 \mathrm{~g}$ dietary fiber $/ \mathrm{kg}$ agar-based diet. The election of an agar-based diet allowed fulfilling the nutrients and part of the water requirement of mice. Details for the preparation of the agar-based diet have been reported elsewhere [59].

[29]. Animals were divided in two treatment groups with $(n=15)$ and without raspberry supplementation $(n=15)$. Obese mice were supplemented with raspberry for 8 weeks whereas control mice were fed with a diet resembling energy content (Tables 1 and S1). Fresh fecal samples were obtained from all animals (control and raspberry-supplemented obese mice) before humane euthanasia at the end of the study (8 weeks) and used for the assessment of bacterial communities.

\section{DNA Extraction}

Total genomic DNA was extracted from all fecal samples using bead-beating and a DNA extraction kit (Zymo Research Corp, USA) according to the manufacturer's protocol. The DNA concentration and purity were assessed using a NanoDrop spectrophotometer (Thermo Scientific, USA). The DNA samples were adjusted to $5 \mathrm{ng} / \mu \mathrm{l}$ and used for high-throughput $16 \mathrm{~S}$ sequencing and quantitative polymerase chain reaction (qPCR) analyses.

\section{High-Throughput 16S rRNA Sequencing for Colonic Microbiota}

The 16S rRNA gene was chosen as a target because this gene contains conserved and variable regions that are useful for studying bacterial communities. In this study, we amplified the V4 region using the primers F515 (5'-GTGCCAGCMGCCGCGGTAA- 
3') and R806 (5'-GGACTACHVGGGTWTCTAAT-3') because this region is the most commonly used in massive surveys of microbial communities and is currently recommended by the Earth Microbiome Project [30]. PCR amplicons were subject to pairedend sequencing using a MiSeq Illumina instrument as described previously [28]. Sequencing was carried out at Molecular Research LP (USA) following the manufacturer's guidelines.

\section{Bioinformatics}

Raw Illumina fastq files were demultiplexed (i.e., assignment of reads to samples), quality filtered, and analyzed using default parameters in split_libraries_fastq.py within the freely available Quantitative Insights into Microbial Ecology (QIIME) Virtual Box ver. 1.8.0 [31]. Operational taxonomic units (OTUs) were assigned using the open reference algorithm published by Rideout et al. [32]. Because massive sequencing efforts often encounter OTUs that are very low abundant, in this study we removed very low abundant OTUs (i.e., OTUs with $<0.005 \%$ sequences) to increase the sensitivity of detecting true phylogroups, as suggested by Navas-Molina et al. [33]. The chimera-checked GreenGenes OTU representative database (ver.13_5) was used as the reference database [34]. In this study, we also assigned OTUs using a closed approach in order to use PICRUSt to predict the functional profile (see below). All sequence data are freely available at the Sequence Read Archive of the NCBI (BioProject: PRJNA415476).

\section{Prediction of Metabolic Profile Using PICRUSt}

Phylogenetic Investigation of Communities by Reconstruction of Unobserved states (PICRUSt [35]) was used to predict the metabolic profile of the bacterial communities. The OTU table from the closed reference approach was normalized for 16S copy numbers and utilized to predict the metagenome in the galaxy platform of PICRUSt ver. 1.1.1 (The Langille Lab, USA). In this study, PICRUSt was used on the full OTU table as well as a filtered OTU table containing a subset of taxa to determine the separate contribution of different bacterial groups to the overall metabolic profile.

\section{qPCR Analysis}

Quantitative real-time PCR analysis was used for a subset of bacterial groups (Table S2) in an effort to confirm the sequencing results as shown elsewhere [36]. The qPCR data were expressed as the log amount of DNA (picograms of amplified DNA) for each bacterial group per $10 \mathrm{ng}$ of total DNA.

\section{Short-Chain Fatty Acid (SCFA) Analysis}

Cecal contents were homogenized with MilliQ water in a proportion of 1:1.5 (w:v) and centrifuged at 12,000 $\times g$ for $10 \mathrm{~min}$. Supernatants were then filtered through a $0.45 \mu \mathrm{m}$ Nylon filter (VWR Syringe Filters; VWR, USA) and analyzed by highperformance liquid chromatography as reported elsewhere [36]. Butyric acid, caproic acid, sodium acetate, sodium propionate, and valeric acid were purchased from VWR (USA) and used as standards to quantify their concentration in the cecal contents as shown elsewhere [36].

\section{mRNA Analysis of Colonic Mucosal Cells}

Colonic mucosal cells scrapped from the terminal colon were collected from all mice and mechanically pulverized in liquid nitrogen. RNA was extracted from these samples using TRIzol LS Reagent as shown elsewhere [36]. The purified mRNA was used to measure several biomarkers of gut health (Table S3).

\section{Proteomics and Metabolomics Analyses of Feces}

The fecal proteome was analyzed using mass spectrometry at the Tissue Imaging and Proteomics Laboratory, and the fecal metabolome was analyzed using gas chromatography time-offlight mass spectrometry at the Laboratory for Cellular Metabolism and Engineering at WSU (see Supplementary Information for all details about these analyses).

\section{Statistical Analyses}

Bacterial abundances were expressed as relative proportions (i.e., percentages of sequences), compared using the Mann-Whitney test in PAST [37], and analyzed using the LDA Effect Size (LEfSe) method [38]. A rarefaction depth of 50,000 (lowest number of sequences per sample) was used for all diversity analyses. The non-parametric Mann-Whitney test was used to compare OTU frequencies between the two treatment groups in QIIME. The nonparametric Adonis and ANOSIM tests were used to investigate whether the grouping of samples was statistically significant, using the compare_categories.py script in QIIME. STAMP [39] was used to analyze the PICRUSt data using Welch's t-test and Bonferroni correction. Principal coordinate analyses on UniFrac distance metrics were used to compare the samples from each treatment group. Additionally, we performed jackknifing (repeatedly resampling a subset of the available data from each sample) to measure the robustness of the clusters. An alpha of 0.05 was used to reject null hypotheses.

Proteomics data were analyzed with Welch approximate t-tests using MeV 4.9.0- MultiExperiment Viewer (http://www.tm4.org/ mev.html). Statistical analyses of the metabolomics were performed on normalized data using MetaboAnalyst 3.0 [40]. Multivariate statistical analysis was further performed with proteomics and metabolomics data. Principal component analysis, an unsupervised technique, was carried out as a first exploration of the data. Partial least-squares discriminant analysis (PLS-DA) analysis, a supervised technique, was carried out to sharpen the discrimination among the treatments according to similar protein/metabolite expression profiles. The variable importance plot (VIP) was used as a formal tool based on the correlation loadings to identify the most relevant proteins/metabolites involved in class distinction. The VIP procedure was then run to select the most important proteins/ metabolites. Only proteins/metabolites that were significant $(p<0.05)$ between experimental groups were used to build the final PLS-DA model using Unscrambler 9.6 (CAMO A/S, Norway). 
Spearman's correlation matrices, featuring variables identified as significant $(p<0.05)$, were performed using R studio 3.4.0.

\section{Results}

Two animals from the supplemented group died for reasons unrelated to the study and all other animals remained clinically healthy throughout the 8-week research period. After open reference OTU picking and removal of very low abundant OTUs, a total of 2.5 million goodquality sequences (average: 272 nucleotides long) were available for analysis. These sequences were assigned to
675 different OTUs using the open approach described above.

\section{Microbial Composition of Samples}

As shown in other studies [36], Firmicutes, Bacteroidetes and Proteobacteria dominated the fecal microbiota of the $\mathrm{db} / \mathrm{db}$ mice (Fig. 1). The most predominant phylum was Firmicutes, accounting for almost half of all sequencing reads (median: $46.5 \%$ across all samples), followed by Bacteroidetes (median: 39.9\%), Proteobacteria (median: $13.7 \%$ ), and others. In this study, raspberry was associated with less Bacteroidetes (median: $37.3 \%$ vs. $43.3 \%$ in control)
A

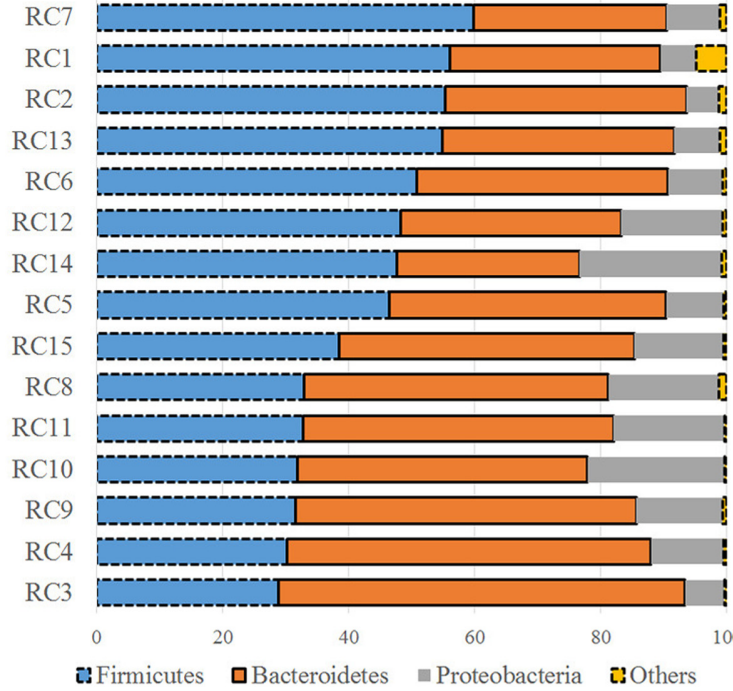

B

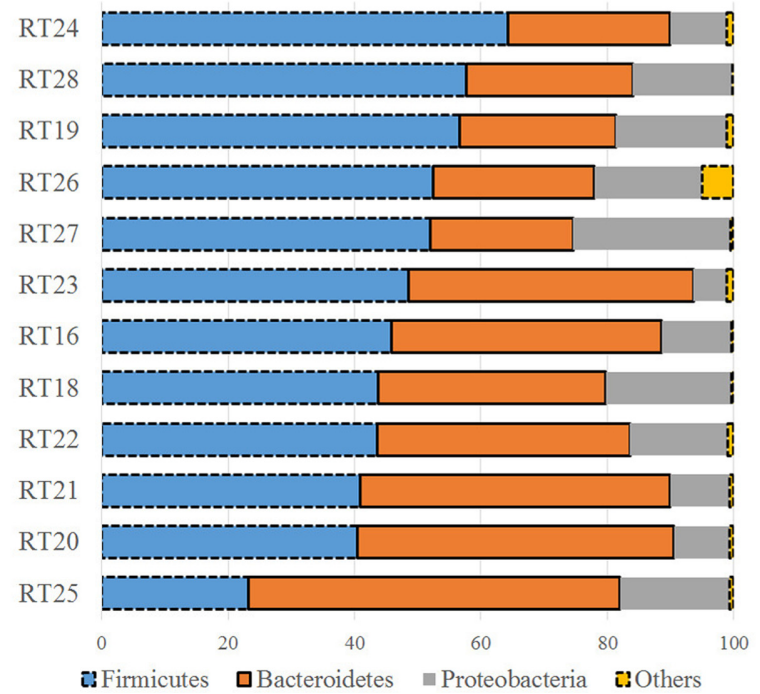

C
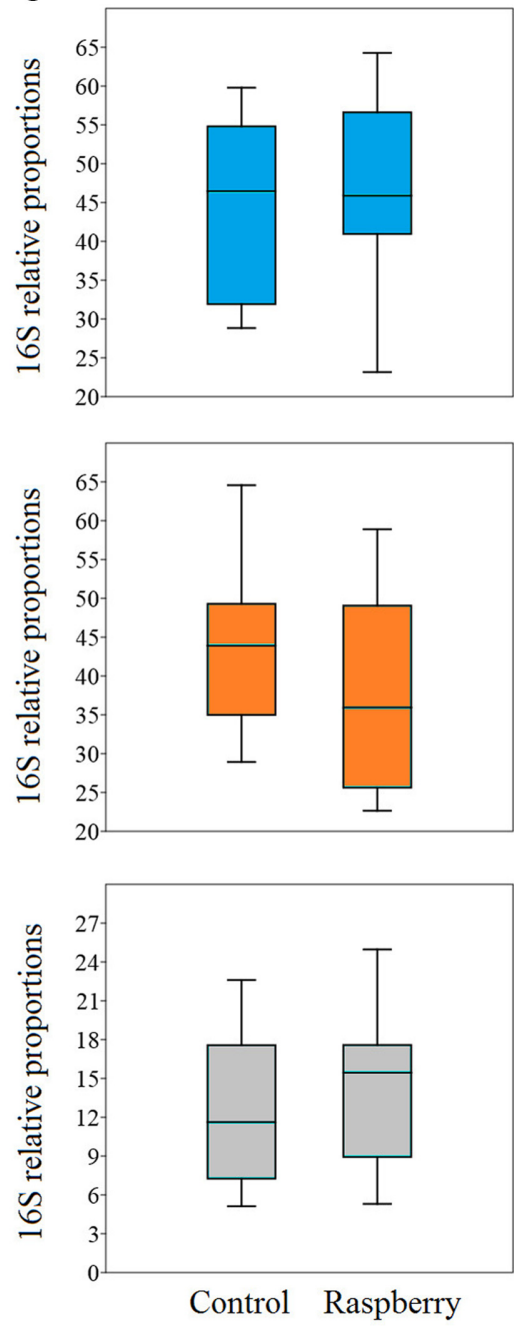

Fig. 1. Bacterial composition of all samples at the phylum level.

(A) Stacked bar charts for the control group. (B) Stacked bar charts for the raspberry-supplemented group. (C) Box plots for the three more abundant phyla (Firmicutes, Bacteroidetes, and Proteobacteria). There was no statistically significant difference in the abundance of any phylum between the treatment groups (see main text for more details). 
A

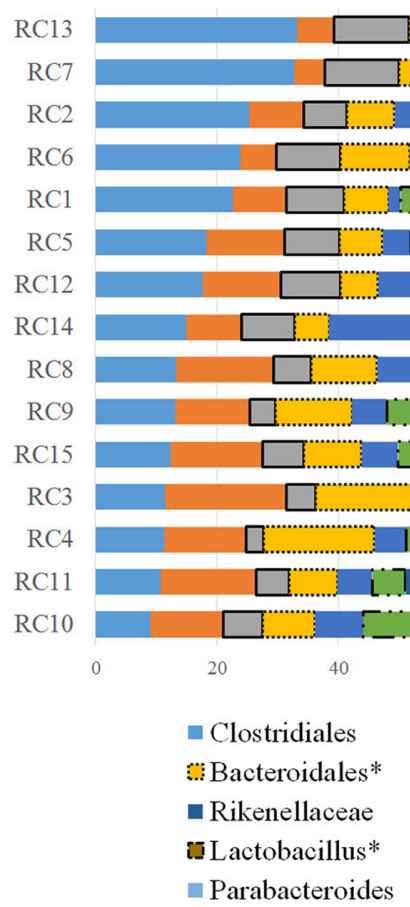

B

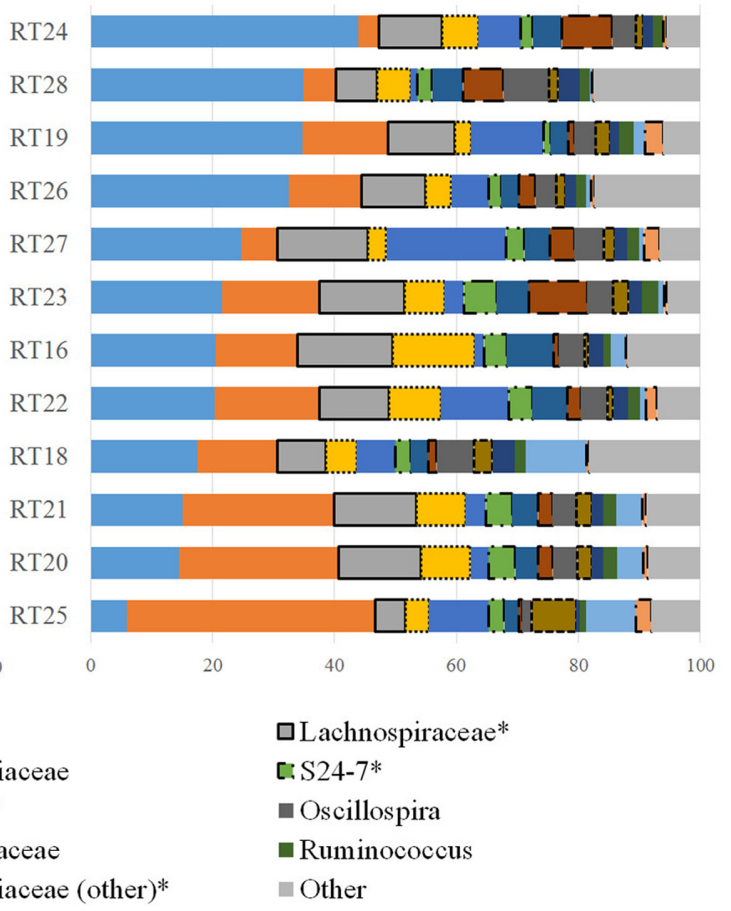

Fig. 2. Bacterial composition of all samples at the genus level.

(A) Stacked bar charts for the control group. (B) Stacked bar charts for the raspberry-supplemented group. An asterisk $\left(^{*}\right)$ denotes statistically significance difference between the two groups. Groups that do not denote a specific genus (e.g., Clostridiales) imply that the taxonomy assignment could not reach the genus level owing to $16 \mathrm{~S}$ rDNA dissimilarity. Please note that Lachnospiraceae was the only group that as a whole was found to be statistically higher in the supplemented group (see main text for more details).

and more Proteobacteria (median: $15.4 \%$ vs. $11.6 \%$ in control), but these differences at the phylum level did not reach statistical significance $(p>0.1)$. Firmicutes remained very similar between the two treatment groups $(\sim 47 \%$, Fig. 1).

Different phyla (e.g., Firmicutes) often contain highly divergent bacterial groups and therefore it is more informative to look at deeper taxonomic levels. Overall, the most abundant group was an unclassified group of Clostridiales (median: 18.2\% across all samples) and the genus Bacteroides (13.0\%) that were not significantly different between the groups $(p=0.11$ and $p=0.29$, respectively) (Fig. 2). The family Lachnospiraceae, however, showed statistically significant difference between the groups $(p=0.009)$, with the supplemented group having higher relative abundance of this group (median: $11.2 \%$ ) than the obese control (median: 7.0\%). Whereas Lachnospiraceae was the only taxa that showed higher abundance in the supplemented group, the comparison of OTU abundances revealed more interesting data (see "Alpha Diversity" below). The higher abundance of Lachnospiraceae in the supplemented mice was accompanied by a lower abundance of an unknown group of Bacteroidales (median: $8.0 \%$ in obese control vs. $5.7 \%$ in raspberry-supplemented, $p=0.02$ ), an unclassified member of the Enterobacteriaceae (median: $2.8 \%$ in control vs. $0.6 \%$ in raspberry, $p=0.005$ ), the family S24-7 (median: $7.9 \%$ in control vs. $2.8 \%$ in supplemented mice, $p=0.0002$ ), the group Odoribacter (median: $6.1 \%$ in control vs. $2.4 \%$ in supplemented group, $p=0.04$ ), and Lactobacillus (median: $4.4 \%$ in control vs. $2.1 \%$ in supplemented mice, $p=0.003$ ) (Fig. 2). Overall these results show that raspberry supplementation is associated with a distinctive fecal bacterial composition characterized by

Table 2. Alpha metrics (median, minimum-maximum).

\begin{tabular}{lccc}
\hline \multicolumn{1}{c}{ Metric } & Control & Raspberry & $p$ Value $^{*}$ \\
\hline Shannon & $6.2(5.6-6.6)$ & $6.4(5.4-6.8)$ & 0.38 \\
Chao1 & $648.7(621-659)$ & $656.1(629-669)$ & 0.08 \\
Species & $610(584-634)$ & $613(552-633)$ & 0.99 \\
PD whole tree & $47.3(46.5-48.5)$ & $47.9(44.2-48.9)$ & 0.32 \\
\hline
\end{tabular}

${ }^{*} p$ values are from the Mann-Whitney test. 
Table 3. Average (min-max) abundance of OTUs that were significantly more abundant between the two treatment groups.

\begin{tabular}{lcccc}
\hline \multicolumn{5}{c}{ OTUs more abundant in supplemented mice } \\
\hline Taxonomy of OTU & Control & Raspberry & Difference between means & $p$ Value $^{*}$ \\
\hline Clostridiales & $113(21-1,146)$ & $1,846(579-4,593)$ & 1,733 & 0.010 \\
Rikenellaceae & $1,248(638-2,529)$ & $2,360(1,020-4,014)$ & 1,112 & 0.043 \\
Clostridiales & $36(13-71)$ & $1,057(92-3,394)$ & 1,021 & 0.031 \\
Bacteroides ovatus & $156(52-335)$ & $970(74-3,704)$ & 814 & 0.016 \\
\hline \multicolumn{7}{r}{} & OTUs more abundant in control mice & & $p$ Value* \\
\hline Taxonomy of OTU & Control & Raspberry & Difference between means & 0.043 \\
S24-7 & 3,027 & 849 & 2,178 & 0.019 \\
Rikenellaceae & 1,084 & 302 & 782 & 0.019 \\
S24-7 & 790 & 126 & 664 & 0.019 \\
S24-7 & 664 & 104 & 560 & \\
\hline
\end{tabular}

${ }^{*} p$ values are from the non-parametric Mann-Whitney test adjusted for false discovery rate in QIIME.

more Lachnospiraceae and less of other bacterial groups.

\section{Alpha Diversity}

We calculated several indices of alpha diversity to investigate whether raspberry supplementation was associated with a difference in the number of OTUs or the OTU diversity in feces. Interestingly, and despite the observed differences in several bacterial groups (e.g., Lachnospiraceae) and community microbial composition (see "Beta Diversity" below), there was no significant difference in the number of
OTUs at the "species" level, the Chao1 metrics (an accurate estimator of the number of different species or richness; Chao [41]), or the Shannon diversity index (Table 2). Although this implies that overall raspberry supplementation is not associated with a different diversity or different types of bacterial organisms, the comparison of OTU frequencies revealed that one OTU related to an unknown member of Clostridiales and one OTU of Rikenellaceae were much more prevalent in supplemented mice, whereas another OTU (also related to Rikenellaceae) was more

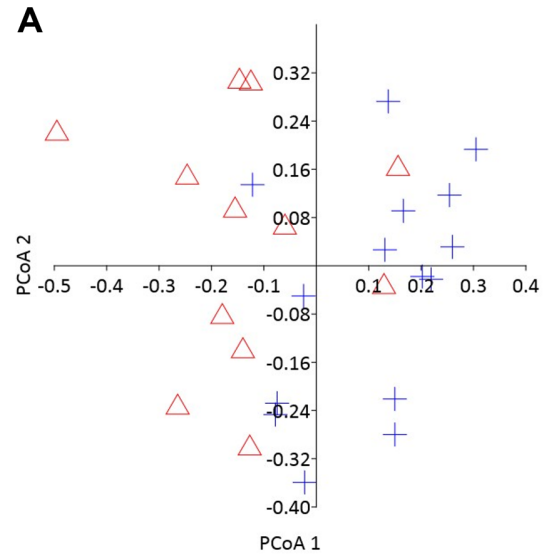

B

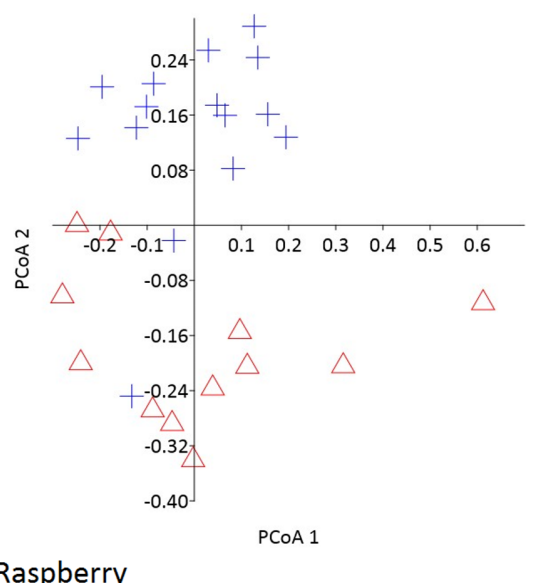

Fig. 3. Principal coordinate analysis plot (PCoA plot) of UniFrac distances.

(A) Weighted UniFrac distances. (B) Unweighted UniFrac distances. The ANOSIM R statistic was 0.42 for weighted and 0.62 for unweighted distances, suggesting that the clustering of samples is stronger when only considering the phylogenetic relationship among the sequences (unweighted UniFrac, B panel). On the other hand, more variation was explained by the grouping using weighted distances (17\%) compared with the variation explained using the unweighted distances (10\%) according to the Adonis test. 
prevalent in control mice (Table 3). This is very interesting because it shows that some members of the same taxonomic group (e.g., Rikenellaceae) may be lower or higher in abundance in a particular treatment group, a phenomenon that has been poorly discussed in the literature.

\section{Beta Diversity}

Although here we only included the variable "treatment" (i.e., with and without raspberry supplementation) to differentiate between the microbial communities, it is noteworthy that a principal coordinate analysis of UniFrac distances revealed statistically significant difference in the bacterial communities between the two groups of mice (Fig. 3, $p<0.001$ for both Adonis and ANOSIM tests), thus confirming that the changes observed at the taxonomic or the OTU level were enough to differentiate both communities. This is important to note because a difference in a few bacterial groups is sometimes not enough to differentiate the whole communities [42]. Jackknifed UPGMA clustering confirmed the strength of the clustering (Fig. 4). Interestingly, beta diversity analyses on the full OTU tables (without removing low abundant OTUs) also revealed significant differences in the composition of microbial communities, thus showing that the observed differences were not biased by the absence of low abundant taxa.

\section{Prediction of Functional Profiles Using PICRUSt}

PICRUSt was used to predict the functional profiles in the fecal microbiota. In this study, we detected a high number of predictive functional features mostly associated with membrane transporters, DNA repair, and recombination proteins. After adjustment for false discovery rate, only two features (ribosome biogenesis and amino acid metabolism) were found to be higher in control mice than in supplemented mice, and this difference almost reached significance ( $p=0.06$ and $p=0.08$, respectively).

PICRUSt using filtered OTU table. The use of filtered OTU tables for specific taxa to predict the metabolic profile using PICRUSt is uncommon in the literature but may shed light into the contribution of different bacterial groups to

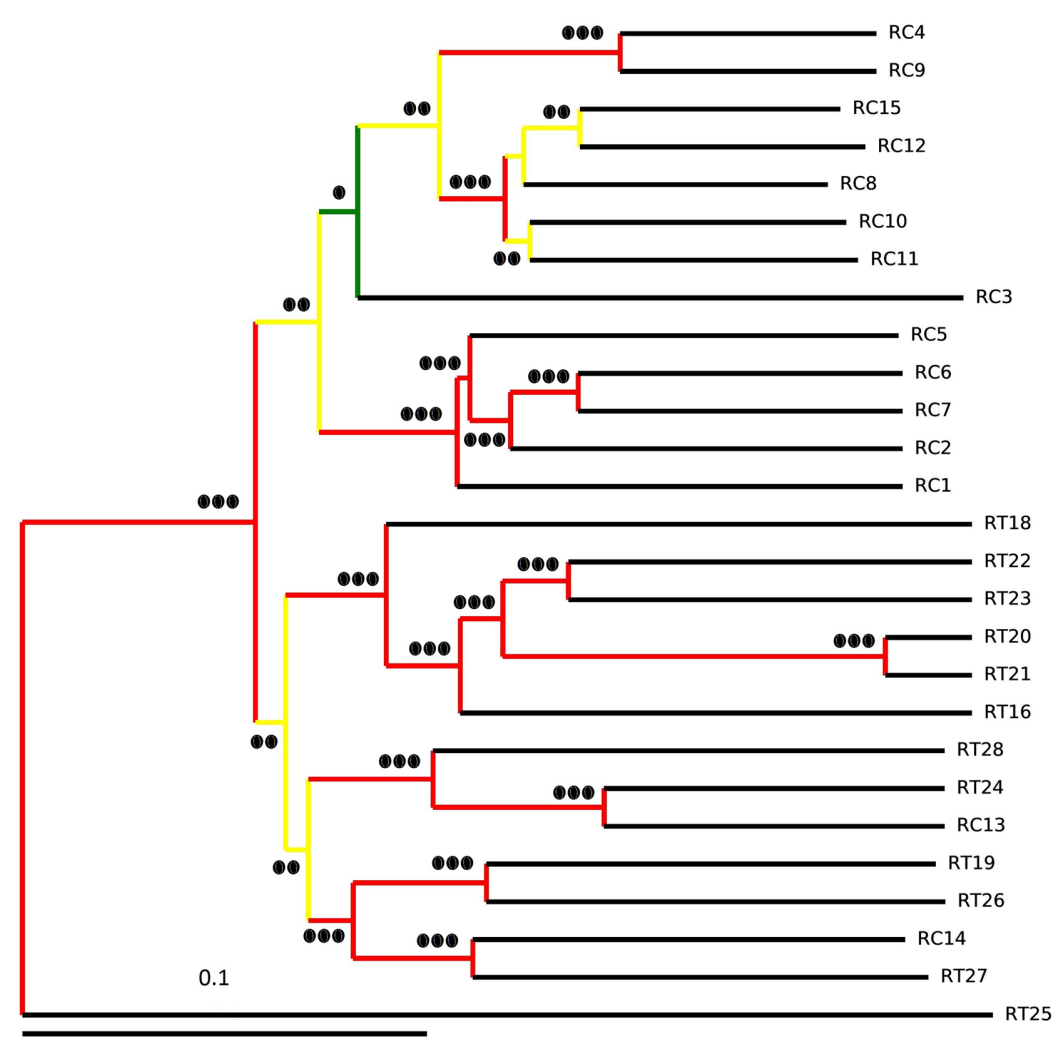

Fig. 4. Jackknifed UPGMA clustering of samples using the weighted UniFrac distance matrix.

RC: control group; RT: raspberry group. Jackknifing was performed using information from 10,000 sequences in each jackknifed subset. Colors represent different jackknife supports: red $(\bullet \bullet \bullet)$ for $75-100 \%$ support, yellow $(\bullet \bullet)$ for $50-75 \%$, and green $(\bullet)$ for $25-50 \%$. The sample RT25 from the raspberry group, which did not cluster together with the rest of the samples from the raspberry group here, had the lowest abundance of Clostridiales and the highest abundance of Bacteroides (see Fig. 2). The bar represents community dissimilarity. 
the overall functional potential [36]. For example, in this study, the separate analysis of OTUs from Lachnospiraceae (the only group that showed higher abundance in supplemented mice) revealed a total of 38 features that showed statistical significance between the groups using the same stringent criteria used for the full OTU tables (e.g., more genes related to lipid metabolism in supplemented mice; see Fig. 5 and Supplementary Information). The separate analysis of other groups such as Bacteroides ovatus, a group that can cause systematic antibody responses [43] and that was more prevalent in the supplemented mice in this study (Table 3), did not yield any difference in the predicted profile between the groups.

\section{qPCR Analyses}

Prevotella $(p=0.0632)$ and the entire family Ruminococcaceae $(p=0.0001)$ were found to be lower in control mice, whereas Bifidobacterium bifidum ( $p=0.0011)$ was lower in supplemented mice. All other groups that were analyzed using qPCR did not show any significant difference. Note that Prevotella and Bifidobacterium were not found in sequencing, and Ruminococcaceae did not show any difference in sequencing. It is not uncommon to find differences between molecular techniques with regard to the abundance of microbial groups.
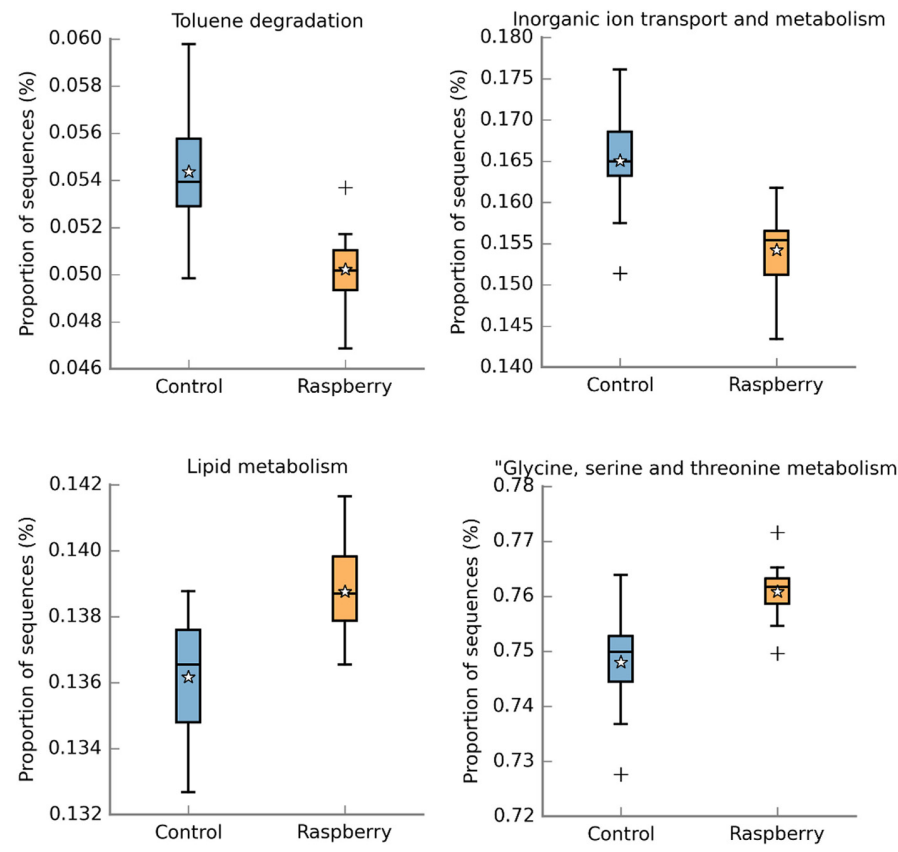

\section{SCFA in Cecal Contents}

Supplemented mice had more butyric acid compared with controls ( 1.3 vs. $0.7 \mathrm{mmol} / \mathrm{mg}$ of cecal content, $p=0.0084$ ). No difference in acetic acid was found and measurements for all other SCFAs showed undetectable levels.

\section{mRNA in Colonic Mucosal Cells}

Results showed that among the biomarkers analyzed in colonic mucosal cells, only IL-1 $\beta$ (an interleukin with strong proinflammatory effect) was significantly downregulated in the raspberry-supplemented group $(p<0.001)$ (Table S4).

\section{Proteomic Profiles in Feces}

Results identified 1,019 proteins expressed by the microbiota in the raspberry and control groups (Fig. S1A). Out of the proteins expressed in both groups, 663 proteins were differentially downregulated in the raspberry group, whereas 229 were differentially downregulated in the control group $(p<0.05)$. The data obtained from PLS-DA discriminated both experimental groups on the basis of 626 proteins (Fig. S1B). These results suggest unique signatures in the host responses driven by the differences in the microbiota colonization. This analysis can complement the results from $16 \mathrm{~S}$ rRNA sequencing analysis and contribute to the understanding of the microbiome activity implications
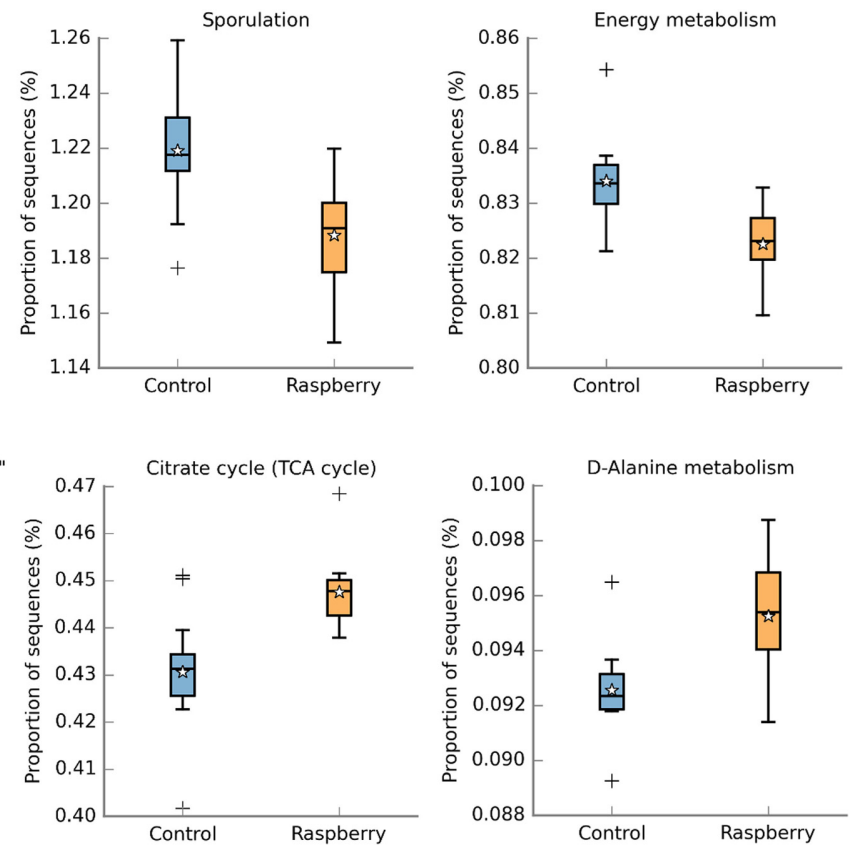

Fig. 5. Box plots showing the relative proportions of sequences associated with PICRUSt features that were significantly different between the treatment groups.

These results were obtained from the analysis of OTUs from Lachnospiraceae only. Please note that PICRUSt results for all OTUs (full OTU table) did not yield any significant difference between the two treatment groups (see main text for details). 
in health and disease-relevant pathways.

\section{Metabolomic Profile}

Results from metabolomic analysis resulted in 139 tentatively identified metabolites in feces of the raspberry and obese control groups. The PLS-DA was able to discriminate samples with a total of explained variance of $36.7 \%$ (Fig. S2) and $42.5 \%$ (Fig. 6A) with two and three factors, respectively. The list of the 25 most important metabolites from the first factor involved in this discrimination is presented in Table S5. Out of the 25 metabolites, 24 were present in higher relative concentrations in the raspberry-supplemented group (serine, alphatocopherol, gamma-aminobutyric, hydrocinnamic acid, sitosterol, alanine, methionine sulfoxide, $\mathrm{N}$-acetyltryptophane, D-glycerol, stigmasterol, tyrosine, mannose, glucose-1phosphate, norleucine, xylose, threonine, glycine, cholestan3-betaol, isoleucine, leucine, valine, glycine-1, oxoproline, and 8-aminocaprylic acid) and only zymosterol 2 was present in higher concentration in obese controls (Fig. S2). Furthermore, a heat map based on $t$-test results $(p<0.05)$ and a false discovery rate cutoff of 0.05 for the 25 top metabolites resulted in eight metabolites that were significant (Fig. 6B).

\section{Discussion}

The gut microbiota is often in symbiosis with the host to ensure mutual survival, but this relationship can sometimes be disturbed, for example during intestinal inflammation or obesity. Although a cause-effect relationship can hardly be established, disorders such as obesity are serious health problems and research aiming to study the effect of different dietary treatments on the obese microbiota is worth pursuing. This study adds valuable information to the literature with regard to the effect of raspberry, a dietary ingredient with beneficial properties, on fecal bacterial communities and metabolites of obese diabetic mice.

Supplementation with raspberry was associated with several changes in the fecal bacterial structure that deserve attention in future studies. For example, Lachnospiraceae was the only bacterial group that, as a whole, was higher in the supplemented group. Lachnospiraceae is a very interesting group that comprises two highly prevalent core phylogroups in humans [44], thus strongly suggesting an important role in maintaining gut homeostasis and health. Also in humans, another study evaluated the effect of a prebiotic, probiotic, or synbiotic on several biomarkers of colorectal cancer and showed that the synbiotic promoted a greater change in microbial communities in part because of a greater proportion of patients harboring fecal Lachnospiraceae [45], thus confirming a potential important role of this group to gut health. Interestingly, Lachnospiraceae is also commonly associated with microbial dysbiosis in canine and feline inflammatory bowel disease, showing less abundance compared with healthy controls [46], and
A

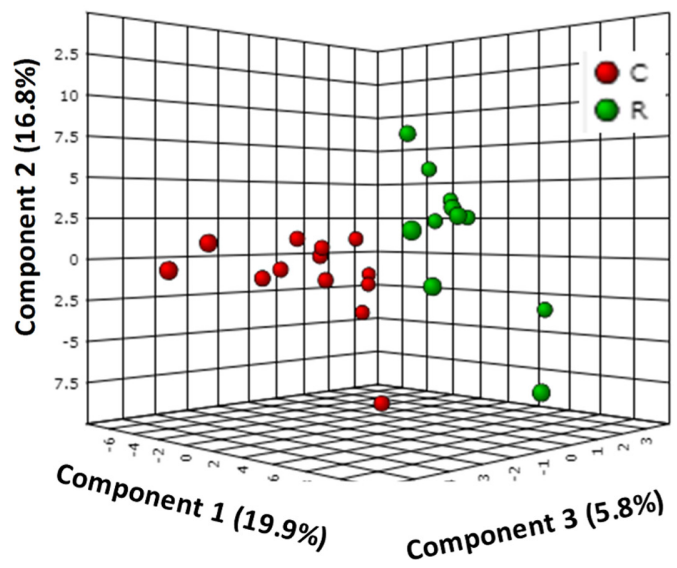

B

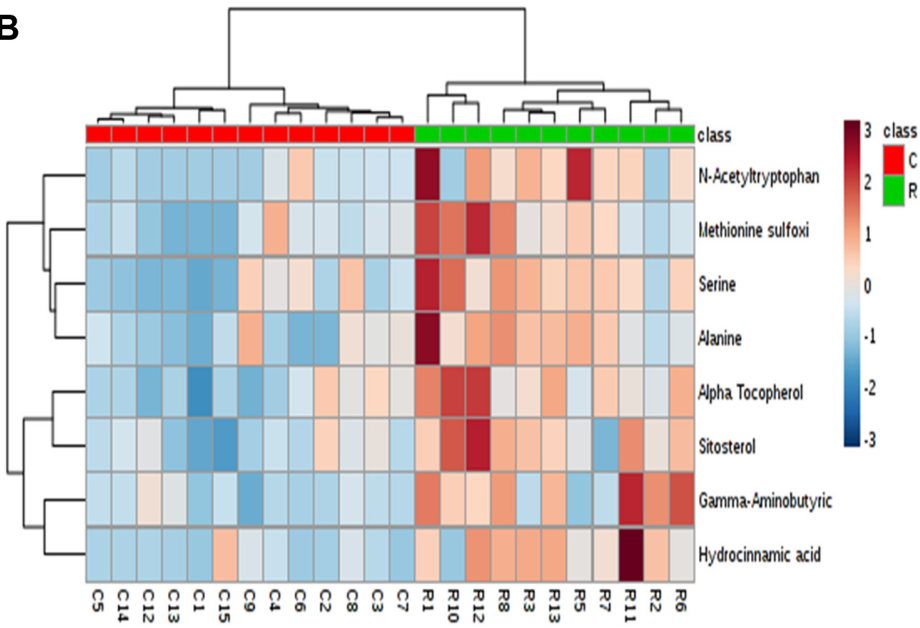

Fig. 6. Raspberry supplementation influenced the composition of fecal metabolites in obese diabetic mice.

(A) Partial least-squares-discriminant analysis with three components that discriminated the obese control (C, red) from the raspberry ( $R$, green) group and explained $42.5 \%$ of the total variance. (B) Hierarchical clustering displaying only the significant metabolites based on a $t$-test $(p<0.05)$ and false discovery rate cutoff $(0.05)$ between the raspberry $(R$, green) and obese control groups $(C$, red). 
inoculation with Lachnospiraceae is associated with decreased Clostridium difficile colonization in mice [47]. All of these findings point to a crucial role of Lachnospiraceae to gut health, and therefore the increase in relative abundance of this group can be considered a beneficial effect associated with the consumption of raspberry in this current study. However, members of the family Lachnospiraceae were found to be more abundant in infants of overweight mothers [48] and in obese subjects [49] and have also been shown to be positively correlated with epididymal adipose tissue [50], thus raising concerns with regard to any potential beneficial effect associated with this bacterial group (please note that we and others have shown that some OTUs may be lower or higher in abundance based on diet, even from the same taxa, thus reflecting the well-known variability at the strain level). It is also important to point out that the results of alpha metrics were unexpected based on the differences in several abundant taxa. We speculate that the higher and lower abundance of certain OTUs (even within the same taxonomic group; see Table 3) contributed to the overall similar abundance in terms of OTU types and numbers.

Other bacterial groups were found to be lower in supplemented mice, such as Lactobacillus, Odoribacter, some unknown groups of Bacteroidales and Enterobacteriaceae, and the S24-7 family (also classified within the Bacteroidales). Importantly, the lower abundance of these groups was not associated with negative side effects in the obese mice. The group S24-7 was also found to be lower in mice fed a highfat diet [51], but other studies have found a higher abundance of this group in obese mice supplemented with wheat [52] and in collagen-induced arthritis-susceptible mice [53]. Interestingly, one recent study administered a Staphylococcus-selective antibiotic to mice for 10 days and only the S24-7 group was reduced at days 6 and 10 of antibiotic administration [54]. The genomes of several members of this S24-7 family have been recently explored, showing that it contains three trophic guilds, each broadly defined by differential abundances of enzymes involved in the degradation of specific carbohydrates (plant, host, and $\alpha$-glucan) [55]. Given the fiber contents in raspberry, and the evidence that fiber consumption may lead to more S247 [36], it remains to be explored the reasons why raspberry would be associated with lower S24-7 in this study. In the future, we expect to see more evidence about the role of this group in gut health and disease.

Data collected from metabolomic analysis of feces supported the prediction of the metabolic profile using PICRUSt regarding a higher relative concentration of metabolites related to lipid metabolism (D-glycerol) and amino acid metabolism (serine, threonine, alanine, etc.). In addition, higher concentrations of phytosterols, gammaaminobutyric acid, and hydroxycinnamic acid derivatives in fecal samples differentiated the supplemented group from the obese controls. This finding is important because it may be related to the beneficial effects associated with raspberry consumption. For instance, phytosterols have been reported to reduce cholesterol absorption from the gut by about 30\%, which leads to lower LDL cholesterol levels in plasma [56]; gamma-aminobutyric acid has important physiological functions, such as neurotransmission, induction of hypotension, as well as diuretic and tranquilizer effects [57]; and hydroxycinnamic acid derivaties have shown potential for the management of lipid metabolism [58]. Overall, our findings point to a series of beneficial consequences to the host that may be overlooked by metabolic predictions.

In general, we have identified a signature of fecal microbiotaproteome-metabolome-host response modulation by raspberry intake that leads to decreased inflammatory IL-1 $\beta$ in colonic mucosal cells. Interestingly, the Spearman correlation analysis (Fig. S3) showed a positive correlation between IL-1 $\beta$ and the Bacteroidales S24-7 bacterial family. Conversely, S24-7 relative abundances were inversely correlated with phytosterols and gamma-aminobutyric acid. The 16S rRNA sequencing analysis has confirmed that S24-7 was less abundant in the supplemented group, but its association with fiber consumption and inflammation is controversial and deserves to be further investigated, particularly in light of new evidence showing that this group is composed by at least three trophic guilds, each specialized in the degradation of different types of carbohydrates [55]. Spearman correlations also helped to identify bacteria that were inversely correlated with amino acid metabolites (genus Anaerobacillus, Lactobacillus, Lactococcus, and Streptococcus) and phytosterols (Enterococcus and Streptococcus) (Fig. S3). In general, our results might lead to develop the necessary efforts to perform omics studies to be able to integrate the data needed to uncover the host pathways that can be modulated by dietary supplementation with raspberry.

This study is not exempt of pitfalls and it is important to discuss them to guide future efforts to study the effect of dietary interventions on the obese microbiota. For instance, whereas other studies have focused on particular ingredients from raspberries (e.g., anthocyanins), in this study we instead addressed the effect of the whole fruit; therefore, the observed effects on the gut microbiota may be the 
simultaneous result of several ingredients (including fiber). More importantly, in this study we did not include lean mice and therefore we could not investigate the relationship between host genetics and the effect of raspberry extracts. Nonetheless, other studies from our research group have shown that interventions with different dietary ingredients yield differences in the fecal microbial structure in obesesupplemented mice that generally do not resemble the obese-control or the lean microbiota [23, 28, 36, 52]. This strongly suggests that the lean microbiota tends to be different when compared with both obese control and supplemented obese mice. Finally, the formulation of the diets was based on isocaloric content and therefore the lower amount of carbohydrates in the control diet (i.e., without raspberry) is indeed related to the particular composition of the fecal microbiota in obese controls (e.g., lower Lachnospiraceae). This is important because, at least in people, raspberries are offered as supplements instead of substitutes of any particular dietary ingredient.

In conclusion, this study shows that supplementation with raspberry extracts is associated with significant changes in the fecal microbiota of obese diabetic mice. Further investigations are needed and are ongoing to study whether these changes are relevant to the treatment of weight disorders in human or veterinary medicine.

\section{Acknowledgments}

The authors would like to express our deepest gratitude to the QIIME and PICRUSt Help Forums for all the support provided. The authors would also like to thank Alejandra Mencia for her technical assistance in the analysis of samples.

\section{Conflict of interest}

This work was supported in part by the National Raspberry Council. The funders had no role in the study design, data collection and analysis, decision to publish, or preparation of the manuscript. Jose F. Garcia-Mazcorro is an employee of MNA de Mexico, and Scot Dowd is an employee of Molecular Research LP.

\section{References}

1. Żukiewicz-Sobczak W, Wróblewska P, Zwoliński J, Chmielewska-Badora J, Adamczuk P, Krasowska E, et al. 2014. Obesity and poverty paradox in developed countries. Ann. Agric. Environ. Med. 21: 590-594.
2. Smith KB, Smith MS. 2016. Obesity statistics. Prim. Care 43: 121-135.

3. Spieker EA, Pyzocha N. 2016. Economic impact of obesity. Prim. Care 43: 83-95.

4. Subhan FB, Chan CB. 2016. Review of dietary practices of the 21st century: facts and fallacies. Can. J. Diabetes 40: 348-354.

5. Clemente JC, Ursell LK, Wegener Parfrey L, Knight R. 2012. The impact of the gut microbiota on human health: an integrative view. Cell 148: 1258-1270.

6. Dinan TG, Cryan JF. 2016. Microbes, immunity, and behavior: psychoneuroimmunology meets the microbiome. Neuropsychopharmacology 42: 178-192.

7. Hill DA, Artis D. 2010. Intestinal bacteria and the regulation of immune cell homeostasis. Annu. Rev. Immunol. 28: 623-667.

8. Harakeh SM, Khan I, Kumosani T, Barbour E, Almasaudi SB, Bahijri SM, et al. 2016. Gut microbiota: a contributing factor to obesity. Front. Cell. Infect. Microbiol. 6: 95.

9. Heiman ML, Greenway FL. 2016. A healthy gastrointestinal microbiome is dependent on dietary diversity. Mol. Metab. 5: $317-320$

10. Wu GD, Chen J, Hoffmann C, Bittinger $K$, Chen Y-Y, Keilbaugh SA, et al. 2011. Linking long-term dietary patterns with gut microbial enterotypes. Science 334: 105-108.

11. Piotr Mazur S, Nes A, Wold AB, Fagertun Remberg S, Aaby K. 2014. Quality and chemical composition of ten red raspberry (Rubus idaeus L.) genotypes during three harvest seasons. Food Chem. 160: 233-240.

12. Burton-Freeman BM, Sandhu AK, Edirisinghe I. 2016. Red raspberries and their bioactive polyphenols: cardiometabolic and neuronal health links. Adv. Nutr. 7: 44-65.

13. McDougall GJ, Stewart D. 2005. The inhibitory effects of berry polyphenols on digestive enzymes. Biofactors 23: 189-195.

14. Noratto GD, Chew BP, Atienza LM. 2017. Red raspberry (Rubus ideaeus L.) intake decreases oxidative stress in obese diabetic $(\mathrm{db} / \mathrm{db})$ mice. Food Chem. 227: 305-314.

15. Overall J, Bonney SA, Wilson M, Beermann A, Grace MH, Esposito D, et al. 2017. Metabolic effects of berries with structurally diverse anthocyanins. Int. J. Mol. Sci. 18: E422.

16. Nowak A, Sójka M, Klewicka E, Lipińska L, Klewicki R, Kolodziejczyk K. 2017. Ellagitannins from Rubus idaeus L. exert geno- and cytotoxic effects against human colon adenocarcinoma cell line Caco-2. J. Agric. Food Chem. DOI: 10.1021/acs.jafc.6b05387.

17. Fotschki B, Juśkiewicz J, Jurgoński A, Rigby N, Sójka M, Kolodziejczyk K, et al. 2017. Raspberry pomace alters cecal microbial activity and reduces secondary bile acids in rats fed a high-fat diet. J. Nutr. Biochem. 46: 13-20.

18. Viladomiu M, Hontecillas R, Lu P, Bassaganya-Riera J. 2013. Preventive and prophylactic mechanisms of action of pomegranate bioactive constituents. Evid. Based Complement. Alternat. Med. 2013: 789764.

19. Zou X, Yan C, Shi Y, Cao K, Xu J, Wang X, et al. 2014. Mitochondrial dysfunction in obesity-associated nonalcoholic 
fatty liver disease: the protective effects of pomegranate with its active component punicalagin. Antioxid. Redox Signal. 21: $1557-1570$.

20. Medjakovic S, Jungbauer A. 2013. Pomegranate: a fruit that ameliorates metabolic syndrome. Food Funct. 4: 19-39.

21. Heber D, Seeram NP, Wyatt H, Henning SM, Zhang $Y$, Ogden LG, et al. 2007. Safety and antioxidant activity of a pomegranate ellagitannin-enriched polyphenol dietary supplement in overweight individuals with increased waist size. J. Agric. Food Chem. 55: 10050-10054.

22. Tomas-Barberan FA, Selma MV, Espin JC. 2016. Interactions of gut microbiota with dietary polyphenols and consequences to human health. Curr. Opin. Clin. Nutr. Metab. Care 19: 471-476.

23. Noratto GD, Garcia-Mazcorro JF, Markel M, Martino HS, Minamoto Y, Steiner JM, et al. 2014. Carbohydrate-free peach (Prunus persica) and plum (Prunus domestica) juice affects fecal microbial ecology in an obese animal model. PLoS One 9: e101723.

24. Lee HC, Jenner AM, Low CS, Lee YK. 2006. Effect of tea phenolics and their aromatic fecal bacterial metabolites on intestinal microbiota. Res. Microbiol. 157: 876-884.

25. Parkar SG, Stevenson DE, Skinner MA. 2008. The potential influence of fruit polyphenols on colonic microflora and human gut health. Int. J. Food Microbiol. 124: 295-298.

26. Bialonska D, Ramnani P, Kasimsetty SG, Muntha KR, Gibson GR, Ferreira D. 2010. The influence of pomegranate by-product and punicalagins on selected groups of human intestinal microbiota. Int. J. Food Microbiol. 140: 175-182.

27. Bolca S, Van de Wiele T, Possemiers S. 2013. Gut metabotypes govern health effects of dietary polyphenols. Curr. Opin. Biotechnol. 24: 220-225.

28. Garcia-Mazcorro JF, Mills DA, Noratto G. 2016. Molecular exploration of fecal microbiome in quinoa-supplemented obese mice. FEMS Microbiol. Ecol. 92: fiw089.

29. Wang B, Chandrasekera PC, Pippin JJ. 2014. Leptin- and leptin receptor-deficient rodent models: relevance for human type 2 diabetes. Curr. Diabetes Rev. 10: 131-145.

30. Caporaso JG, Lauber CL, Walters WA, Berg-Lyons D, Huntley J, Fierer N, et al. 2012. Ultra-high-throughput microbial community analysis on the Illumina HiSeq and MiSeq platforms. ISME J. 6: 1621-1624.

31. Caporaso JG, Kuczynski J, Stombaugh J, Bittinger K, Bushman FD, Costello EK, et al. 2010. QIIME allows analysis of high-throughput community sequencing data. Nat. Methods 7: 335-336.

32. Rideout JR, He Y, Navas-Molina JA, Walters WA, Ursell LK, Gibbons SM, et al. 2014. Subsampled open-reference clustering creates consistent, comprehensive OTU definitions and scales to billions of sequences. PeerJ 2: e545.

33. Navas-Molina JA, Peralta-Sanchez JM, Gonzalez A, McMurdie PJ, Vazquez-Baeza Y, Xu Z, et al. 2013. Advancing our understanding of the human microbiome using QIIME. Methods Enzymol. 531: 371-444.
34. DeSantis TZ, Hugenholtz P, Larsen N, Rojas M, Brodie EL, Keller K, et al. 2006. Greengenes, a chimera-checked $16 \mathrm{~S}$ rRNA gene database and workbench compatible with ARB. Appl. Environ. Microbiol. 72: 5069-5072.

35. Langille MG, Zaneveld J, Caporaso JG, McDonald D, Knights D, Reyes JA, et al. 2013. Predictive functional profiling of microbial communities using 16S rRNA marker gene sequences. Nat. Biotechnol. 31: 814-821.

36. Garcia-Mazcorro JF, Nunes Lage N, Mertens-Talcott S, Talcott S, Chew B, Dowd SE, et al. 2017. Effect of dark sweet cherry powder consumption on the gut microbiota, shortchain fatty acids, and biomarkers of gut health in obese $\mathrm{db} /$ db mice. PeerJ 6: e4195.

37. Hammer $\varnothing$, Harper DAT, Ryan PD. 2001. PAST: paleontological statistics software package for education and data analysis. Paleontol. Electron. 4: 1-9.

38. Segata N, Izard J, Waldron L, Gevers D, Miropolsky L, Garrett WS, Huttenhower C. 2011. Metagenomic biomarker discovery and explanation. Genome Biol. 12: R60.

39. Parks DH, Beiko RG. 2010. Identifying biologically relevant differences between metagenomic communities. Bioinformatics 26: 715-721.

40. Xia J, Sinelnikov IV, Han B, Wishart DS. 2015. MetaboAnalyst 3.0 - making metabolomics more meaningful. Nucleic Acids Res. 43: W251-W257.

41. Chao A. 1984. Nonparametric estimation of the number of classes in a population. Scand. J. Stat. 11: 265-270.

42. Garcia-Mazcorro JF, Castillo-Carranza SA, Guard B, GomezVazquez JP, Dowd SE, Brightsmith DJ. 2017. Comprehensive molecular characterization of bacterial communities in feces of pet birds using $16 \mathrm{~S}$ marker sequencing. Microb. Ecol. 73: 224-235.

43. Saitoh S, Noda S, Aiba Y, Takagi A, Sakamoto M, Benno Y, Koga Y. 2002. Bacteroides ovatus as the predominant commensal intestinal microbe causing a systemic antibody response in inflammatory bowel disease. Clin. Diagn. Lab. Immunol. 9: 54-59.

44. Sekelja M, Berget I, Næs T, Rudi K. 2011. Unveiling an abundant core microbiota in the human adult colon by a phylogroup-independent searching approach. ISME J. 5: 519-531.

45. Worthlet DL, Le Leu RK, Whitehall VL, Conlon M, Christophersen C, Belobrajdic D, et al. 2009. A human, double-blind, placebo-controlled, crossover trial of prebiotic, probiotic, and synbiotic supplementation: effects on luminal, inflammatory, epigenetic, and epithelial biomarkers of colorectal cancer. Am. J. Clin. Nutr. 90: 578-586.

46. Suchodolski JS. 2011. Companion animals symposium: microbes and gastrointestinal health of dogs and cats. $J$. Anim. Sci. 89: 1520-1530.

47. Reeves AE, Koenigsknecht MJ, Bergin IL, Young VB. 2012. Suppression of Clostridium difficile in the gastrointestinal tracts of germfree mice inoculated with a murine isolate from 
the family Lachnospiraceae. Infect. Immun. 80: 3786-3794.

48. Tun HM, Bridgman SL, Chari R, Field CJ, Guttman DS, Becker AB, et al. 2018. Roles of birth mode and infant gut microbiota in intergenerational transmission of overweight and obesity from mother to offspring. JAMA Pediatr. 172: 368-377.

49. de la Cuesta-Zuluaga J, Corrales-Agudelo V, Carmona JA, Abad JM, Escobar JS. 2018. Body size phenotypes comprehensively assess cardiometabolic risk and refine the association between obesity and gut microbiota. Int. J. Obes. (Lond.) 42: 424-432.

50. Higashimura Y, Baba Y, Inoue R, Takagi T, Mizushima K, Ohnogi $\mathrm{H}$, et al. 2017. Agaro-oligosaccharides regulate gut microbiota and adipose tissue accumulation in mice. J. Nutr. Sci. Vitaminol. (Tokyo) 63: 269-276.

51. Tomas J, Mulet C, Saffarian A, Cavin JB, Ducroc JB, Regnault B, et al. 2016. High-fat diet modifies the PPAR- $\gamma$ pathway leading to disruption of microbial and physiological ecosystem in murine small intestine. Proc. Natl. Acad. Sci. USA 113: E5934-E5943.

52. Garcia-Mazcorro JF, Ivanov I, Mills DA, Noratto G. 2016. Influence of whole-wheat consumption on fecal microbial ecology of obese diabetic mice. PeerJ 4: e1702.
53. Liu X, Zeng B, Zhang J, Li W, Mou F, Wang H, et al. 2016. Role of the gut microbiome in modulating arthritis progression in mice. Sci. Rep. 6: 30594.

54. Yao J, Carter RA, Vuagniaux G, Barbier M, Rosch JW, Rock CO. 2016. A pathogen-selective antibiotic minimizes disturbance to the microbiome. Antimicrob. Agents Chemother. 60: 4264-4273.

55. Ormerod KL, Wood DL, Lachner N, Gellatly SL, Daly JN, Parsons JD, et al. 2016. Genomic characterization of the uncultured Bacteroidales family S24-7 inhabiting the guts of homeothermic animals. Microbiome 4: 36.

56. Marangoni F, Poli A. 2010. Phytosterols and cardiovascular health. Pharmacol. Res. 61: 193-199.

57. Li H, Cao Y. 2010. Lactic acid bacterial cell factories for gamma-aminobutyric acid. Amino Acids 39: 1107-1116.

58. Alam MA, Subhan N, Hossain H, Hossain M, Reza HM, Rahman MM, et al. 2016. Hydroxycinnamic acid derivatives: a potential class of natural compounds for the management of lipid metabolism and obesity. Nutr. Metab. (Lond). 13: 27.

59. Noratto G, Chew BP, Ivanov I. 2016. Red raspberry decreases heart biomarkers of cardiac remodeling associated with oxidative and inflammatory stress in obese diabetic db/db mice. Food Funct. 7: 4944-4955. 